\title{
Percutaneous pulmonary artery venting via jugular vein while on peripheral extracorporeal membrane oxygenation running: a less invasive approach to provide full biventricular unloading
}

\author{
Antonio Loforte ${ }^{1}$, Massimo Baiocchi ${ }^{1}$, Gregorio Gliozzi ${ }^{1}$, Giuditta Coppola ${ }^{1}$, Roberto Di Bartolomeo ${ }^{1}$, \\ Roberto Lorusso $^{2}$ \\ ${ }^{1}$ Department of Cardiothoracic, Transplantation and Vascular Surgery, S. Orsola Hospital, Bologna University, Bologna, Italy; ${ }^{2}$ Cardio-Thoracic \\ Surgery Department, Heart \& Vascular Centre, Maastricht University Medical Centre, Maastricht, The Netherlands \\ Correspondence to: Dr. Antonio Loforte, MD, PhD. Department of Cardiothoracic, Transplantation and Vascular Surgery, S. Orsola Hospital, ALMA \\ Mater Studiorum University of Bologna, Via Massarenti n.9, 40138 Bologna, Italy. Email: antonioloforte@yahoo.it.
}

Submitted Jun 15, 2018. Accepted for publication Aug 13, 2018.

doi: $10.21037 /$ acs.2018.08.06

View this article at: http://dx.doi.org/10.21037/acs.2018.08.06

\section{Introduction}

A delicate concern while on peripheral veno-arterial (V-A) extracorporeal membrane oxygenation (ECMO) therapy is the retrograde flow in the aorta towards the left ventricle (LV), with increase of $L V$ afterload (1-5). The consequent LV pressure overload may account for LV dilatation, increase in left atrial (LA) pressure and pulmonary edema. Furthermore, LV overload increases the wall stress, thus negative influencing the process of myocardial function recovery. If the overload is extreme and $\mathrm{LV}$ contractile impairment significant, the aortic valve may remain closed permanently, causing blood stasis and predisposing thrombi formation in the left heart chambers. In this manuscript, we describe our institutional approach for LV decompression while on peripheral ECMO running by percutaneously venting the pulmonary artery $(\mathrm{PA})$ trunk.

\section{Clinical vignette}

A 59-year-old man was admitted at our institution with a clinical diagnosis of cardiogenic shock due to a recent extensive anterior acute myocardial infarction (AMI) despite early multivessel percutaneous transcatheter angioplasty (PTCA) treatment. A transthoracic echocardiography showed a dilated and hypokinetic $\mathrm{LV}$ with an ejection fraction of $10 \%$ and revealed an intraventricular thrombus adherent to the apex and the anterior wall of LV (Figure 1). The haemodynamics were poor, despite percutaneous insertion of an intra-aortic balloon pump (IABP) support.

\section{Surgical technique}

Preparation

After a multidisciplinary CS team discussion, it was decided to proceed with femoro-axillary V-A ECMO placement associated with percutaneous $L V$ venting, by usage of a BioMedicus NextGen (Medtronic Inc., Minneapolis, MN, USA) cannula via right internal jugular (IJ) vein access to reach and drain the main PA. The fluoroscopy was needed for pulmonary cannula insertion and the hybrid operating room (OR) was judged be the correct location for appropriate cannulae placement. The Permanent Life Support (PLS) (Getinge, Permanent Life Support, Maquet, Jostra Medizintechnik AG, Hirrlingen, Germany) ECMO circuit was, as routinely, primed and adapted for the Levitronix CentriMag (Abbott, Levitronix LLC, Waltham, MA, USA) (1).

\section{Operation (see Video 1)}

The ECMO circuit lines are held and fixed on-table. The tubings are interrupted and prepared for further cannulae connection. The right axillary artery is prepared after 
cut-down and surgical vessel exposure. A heparin drip is delivered. The femoral vein cannulation is performed percutaneously (Figure 2), on the left groin, via Seldinger technique by usage of a DLP Bio-Medicus (Medtronic) cannula (21 Fr, venous drainage). After de-airing, the cannula is connected to a ' $y$ ' line of the ECMO circuit for venous drainage. The axillary artery cannulation is performed, similarly, via Seldinger technique and same BioMedicus cannula (19 Fr). After tunnelling through the skin (Figure 2), the cannula is connected to the arterial line of the ECMO circuit.

A right IJ venous access is established using direct ultrasound visualization. A Lunderquist guidewire (Cook Inc., Bloomington, IL, USA) is advanced under fluoroscopic guidance into the right atrium. A $15 \mathrm{Fr}(50 \mathrm{~cm}$

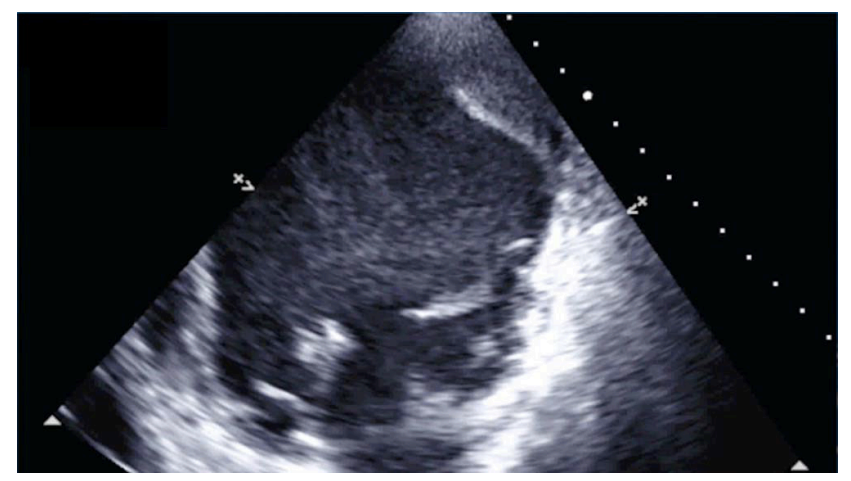

Figure 1 Transthoracic echocardiography showing a dilated LV with an intraventricular thrombus adherent to the apex and the anterior wall of $\mathrm{LV}$. $\mathrm{LV}$, left ventricle. in length) Bio-Medicus NextGen (Medtronic) cannula is then advanced over the Lunderquist wire with its distal tip positioned at the level of tricuspid valve. The Lunderquist wire is removed and a Swan-Ganz catheter (Edwards Lifesciences, Irvine, CA, USA) is advanced into the BioMedicus cannula with its distal tip positioned in the main PA. The cannula is then advanced over the Swan-Ganz catheter to get the PA too (Figure 3). The Swan-Ganz catheter is removed and the cannula, after de-airing, is connected through a ' $y$ ' line to the venous drainage of the ECMO circuit.

The PLS with Quadrox D oxygenator (Getinge, Maquet) circuit is now fully connected to the cannualae and the Levitronix CentriMag (Abbott, Levitronix LLC, Waltham, MA, USA) pump system is now ready for running to further stabilize the haemodynamics.

\section{Postoperative outcome}

Peripheral V-A ECMO support time was 10 days. The patient was successfully weaned from ECMO since improved LV contractility (ejection fraction, 35-40\%) and the system easily removed in the intensive care unit. No ECMO related adverse events occurred and the LV thrombus progressively reduced in size to being not visible at discharge.

\section{Comments}

The most important concern in the current widespread use of peripheral V-A ECMO therapy is the high risk to develop
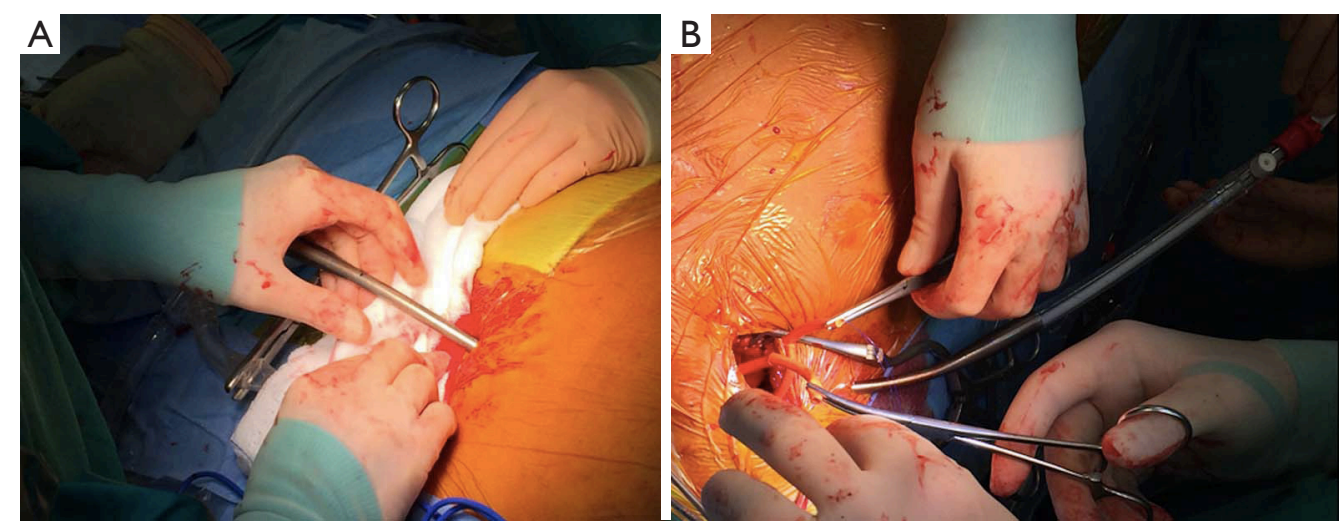

Figure 2 (A) Percutaneous femoral vein cannulation, via Seldinger technique by usage of a Bio-Medicus NextGen (Medtronic) cannula. (B) Axillary artery cannulation, after surgical vessel exposure, via Seldinger technique by usage of a Bio-Medicus NextGen (Medtronic) cannula which is thereafter tunnelled through the skin. 

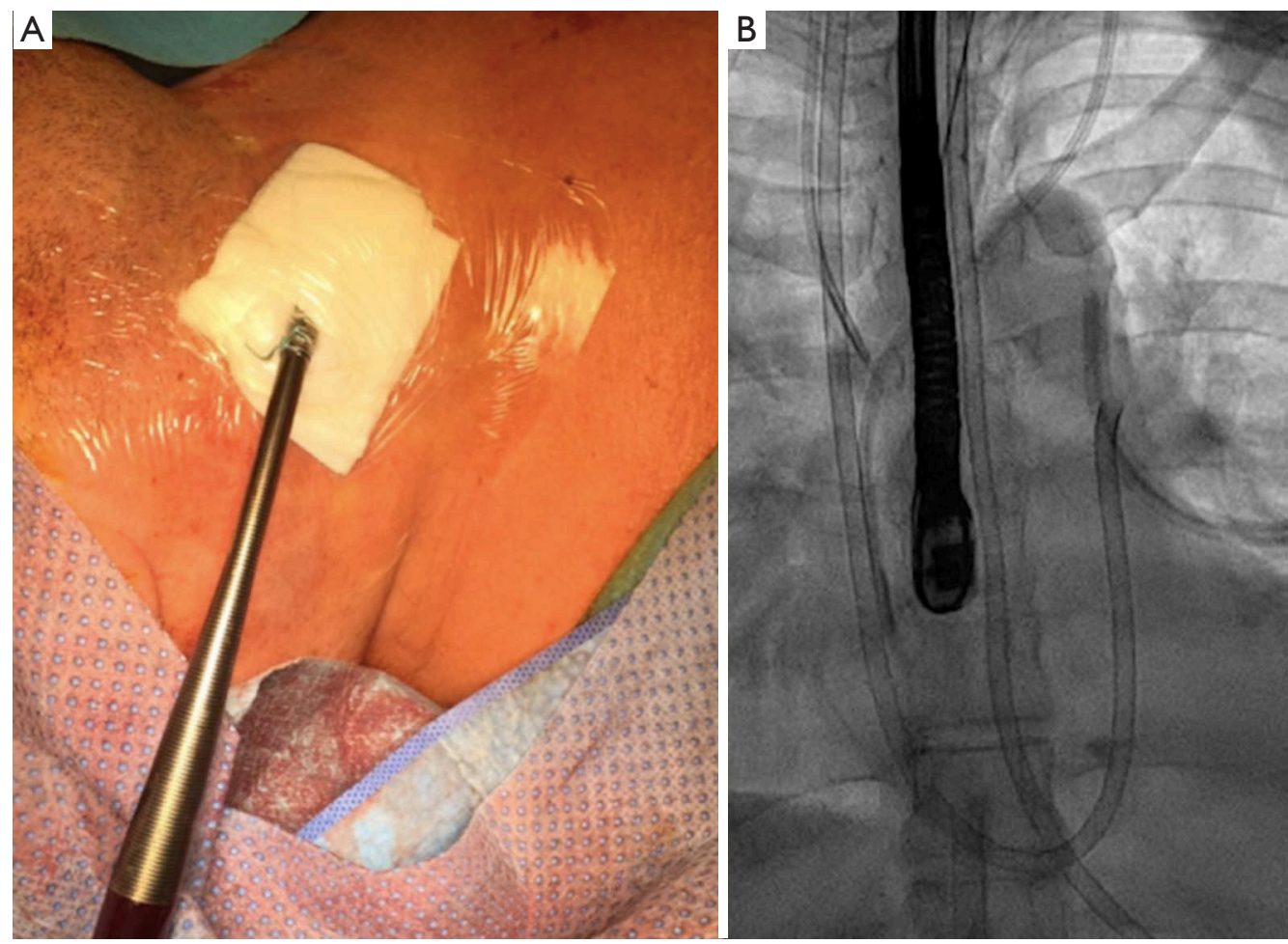

Figure 3 Percutaneous PA cannulation (Bio-Medicus NextGen, Medtronic) via right IJ venous access (A, surgical view; B, fluoroscopic view). IJ, internal jugular; PA, pulmonary artery.

severe LV distension, which compromises myocardial function recovery and leads to pulmonary edema (1-5).

In case of not recommended usage of direct $\mathrm{LV}$ apical venting (e.g., LV apical thrombi, recent antero-apical AMI, acute myocarditis and graft failure after heart transplantation), we institutionally prefer the adoption of $\mathrm{V}$-A peripheral ECMO support associated with a PA drainage, since it enables the rapid onset of extracorporeal life support with an effective biventricular unloading. The procedure is performed percutaneously, without the need for surgical manipulation, with the associated risk for bleeding, heart manipulation and heart injury and it is less expensive than the application of axial pumps, in addition to being easier and faster than all conventional methods.

Fluoroscopy is needed for pulmonary cannula insertion. The hybrid OR may be the correct location for appropriate cannulae placement, which was the case for all our patients. The usage of a Swan-Ganz catheter as a guide for pulmonary cannula advancement may be less traumatic and more confident for the operator who is usually represented by an anesthesiologist or an interventional cardiologist.

\section{Acknowledgements}

None.

\section{Footnote}

Conflicts of Interest: The authors have no conflicts of interest to declare.

\section{References}

1. Loforte A, Marinelli G, Musumeci F, et al. Extracorporeal membrane oxygenation support in refractory cardiogenic shock: treatment strategies and analysis of risk factors. Artif Organs 2014;38:E129-41.

2. von Segesser LK, Kwang K, Tozzi P, et al. A simple way to decompress the left ventricle during venoarterial bypass. Thorac Cardiovasc Surg 2008;56:337-41.

3. Rupprecht L, Flörchinger B, Schopka S, et al. Cardiac decompression on extracorporeal life support: a review and discussion of the literature. ASAIO J 2013;59:547-53. 
4. Meani P, Gelsomino S, Natour E, et al. Modalities and Effects of Left Ventricle Unloading on Extracorporeal Life support: a Review of the Current Literature. Eur J Heart Fail 2017;19 Suppl 2:84-91.

Cite this article as: Loforte A, Baiocchi M, Gliozzi G, Coppola G, Di Bartolomeo R, Lorusso R. Percutaneous pulmonary artery venting via jugular vein while on peripheral extracorporeal membrane oxygenation running: a less invasive approach to provide full biventricular unloading. Ann Cardiothorac Surg 2019;8(1):163-166. doi: 10.21037/ acs.2018.08.06
5. Lorusso R. Are two crutches better than one? The ongoing dilemma on the effects and need for left ventricular unloading during veno-arterial extracorporeal membrane oxygenation. Eur J Heart Fail 2017;19:413-5. 